\title{
RF DESIGN AND PROCESSING OF A POWER COUPLER FOR THIRD HARMONIC SUPERCONDUCTING CAVITIES*
}

\author{
Jianjian Li, Elvin Harms, Tom Kubicki, Dennis Nicklaus, Daniel Olis, Peter Prieto, John Reid, \\ Nikolay Solyak", FNAL, Batavia, IL 60510, U.S.A. \\ Thomas Wong, IIT, Chicago, IL 60616, U.S.A.
}

\section{Abstract}

The FLASH user facility providing free electron laser radiation is built based on the TTF project at DESY. Fermilab has the responsibility for the design and processing of a third harmonic, $3.9 \mathrm{GHz}$, superconducting cavity which is powered via a coaxial power coupler. Six power couplers have been manufactured at CPI after successful design of the power coupler including RF simulation, multipacting calculation, and thermal analysis. The power couplers are being tested and processed with high pulsed power in an elaborate test stand at Fermilab now. This paper presents the RF design and processing work of the power coupler.

\section{INTRODUCTION}

The third harmonic superconducting cavities operating at the frequency of $3.9 \mathrm{GHz}$ have been proposed to increase the peak beam current and to compensate for the non-linear distortions in the longitudinal phase space due to the RF curvature of the $1.3 \mathrm{GHz}$ cavity accelerating voltage. Installation of the third harmonic cavity will allow us to generate ultra-short and highly charged beam bunches with an extremely small transverse emittance [1]. The maximum electron population will be increased by more than $300 \%$ according to the theoretical estimation, as shown in Fig. 1.

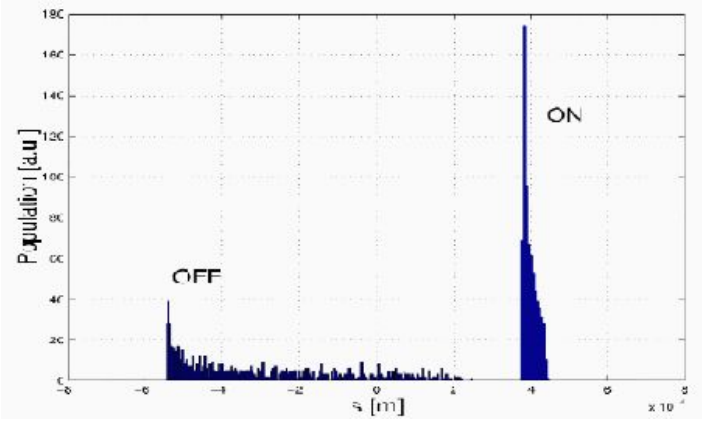

Figure 1: Electron population in a beam bunch, downstream of the bunch compressor, with and without the third harmonic system (calculated by K. Floettmann, DESY, Germany).

\section{RF DESIGN AND OPTIMIZATION}

A completely new power coupler has been developed at Fermilab after successful design of the power coupler including RF simulation and optimization, multipacting calculation, and thermal analysis [2]. Layout of the final

*Work supported by U.S. Department of Energy.

\#solyak@fnal.gov power coupler is shown in Fig. 2. All components of the power coupler: cold and warm windows, bellows section, waveguide-to-coax transition, vacuum and diagnostic ports were optimized for low power reflection at the operating frequency. The field distributions of the entire power coupler are shown in Fig. 3. Windows are located in the positions with very low electric and magnetic fields to reduce the risk of voltage breakdown and heating problem. The power reflection coefficient shown in Fig. 4 is reduced to $-21 \mathrm{~dB}$ at the operating frequency after a series of parameter optimizations.

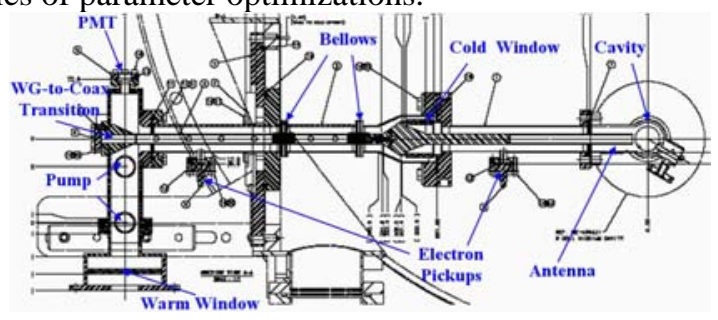

Figure 2: Layout of the $3.9 \mathrm{GHz}$ power coupler.

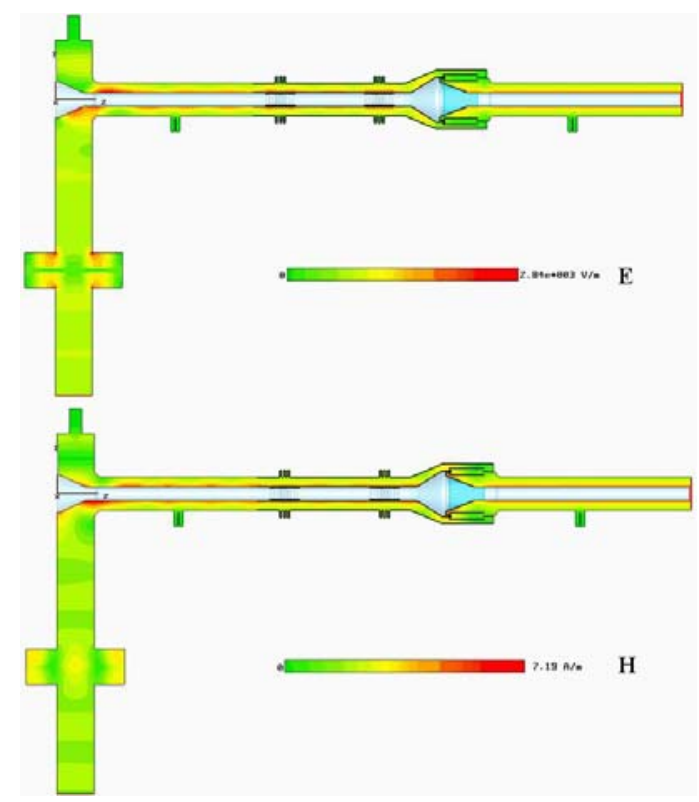

Figure 3: Field distributions of the power coupler.

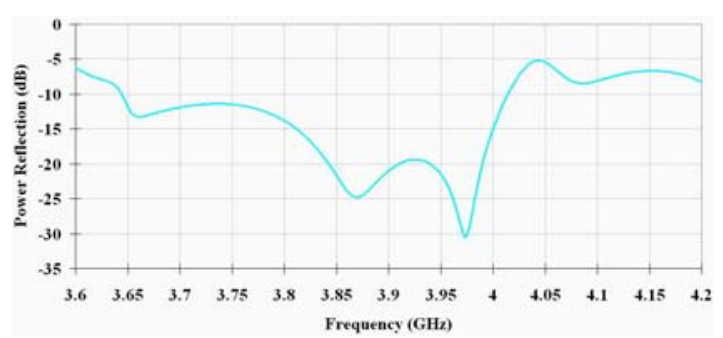

Figure 4: Power reflection coefficient versus frequency. 
The power couplers are being tested in a test stand shown in Fig. 5 for high power processing. Two couplers are assembled with their antennas connected through a waveguide transition. Our design can enable the maximum power transfer between the couplers in the waveguide. The whole processing must include adequate protection to prevent vacuum leaking, windows overheating, and arcing or sparking phenomenon. The couplers in this design have been equipped with this kind of sensors including vacuum gauges, electron pickups, PMT, and RTD.

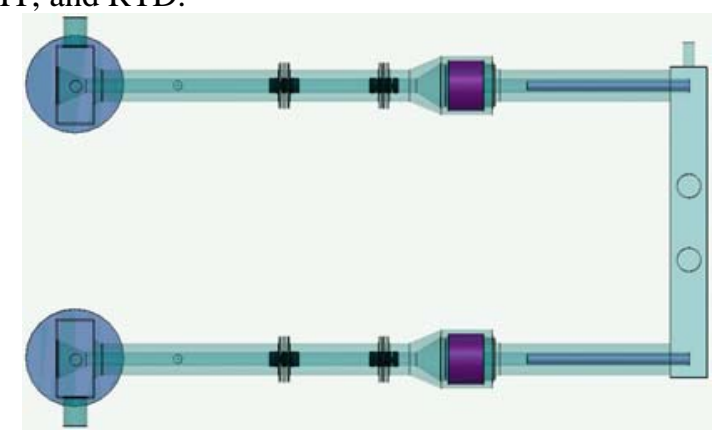

Figure 5: Model of the coupler test stand.

\section{RF PROCESSING PROCEDURE AND RESULTS}

We have fabricated six power couplers from CPI. At first, some parts of the power couplers did not pass the quality test due to the leaking problems or copper plating defects. After being repaired, all components including cold assemblies, warm assemblies, and outer conductors were returned to Fermilab and ready for high power processing.

Table 1: High power processing procedure.

\begin{tabular}{|c|c|}
\hline \multicolumn{2}{|c|}{ High power processing procedure } \\
\hline Pulse Length ( $\mu S$ ) & 20501002004008001300 \\
\hline Rep. Rate (Hz): & 125 \\
\hline Wave Mode & Traveling Wave \\
\hline Peak Power $(\mathrm{kW})$ & Max. 80 \\
\hline Number of Sensors & $\begin{array}{l}4 \text { electron pickups } \\
3 \text { photo detectors } \\
2 \text { temperature detectors } \\
3 \text { vacuum gauges }\end{array}$ \\
\hline
\end{tabular}

The first two power couplers have been assembled in the test stand for high power processing, as shown in Fig. 6. $80 \mathrm{~kW}$ klystron and modulator are also pictured in Fig. 7. Before processing the whole assembly was thoroughly cleaned in an ultrasonic bath and baked at $150{ }^{\circ} \mathrm{C}$ for one week to remove the impurities. The processing is usually done at traveling wave and under room temperature environment. The power is cycled from low to high levels, starting with short pulses ( $20 \mu \mathrm{s}$ ). After reaching the utmost power limit of the klystron the pulse length is doubled. This procedure will be repeated until the full pulse length of $1300 \mu \mathrm{s}$ is reached [3], as shown in Table 1.

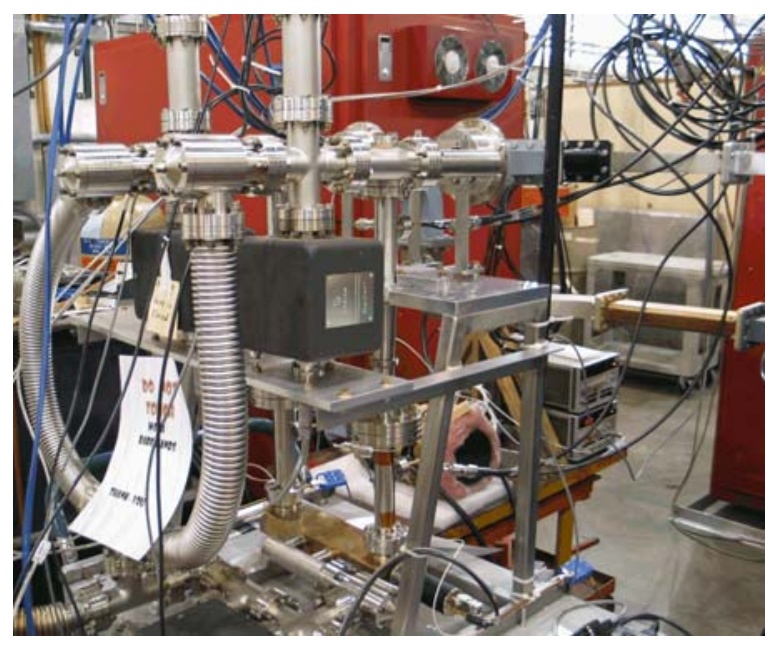

Figure 6: The coupler test stand equipped with vacuum and diagnostic devices.

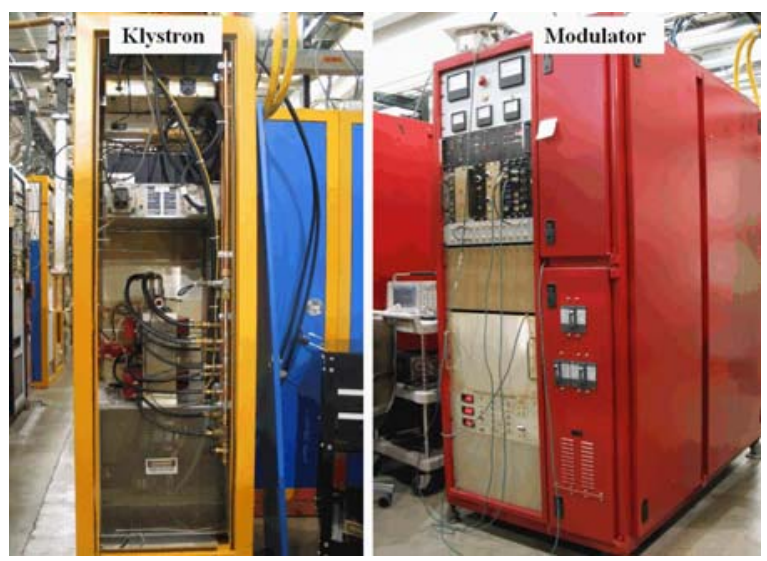

Figure 7: $80 \mathrm{~kW}$ klystron and the corresponding modulator.

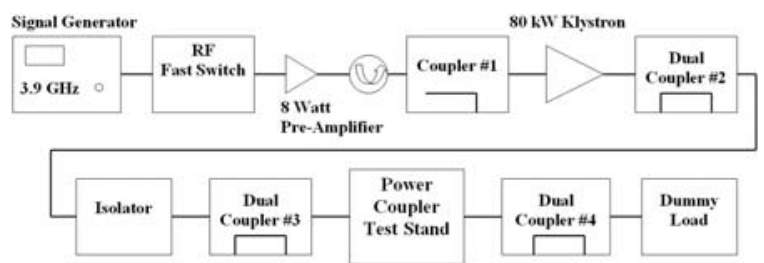

Figure 8: RF system diagram for high power processing.

RF system diagram for high power processing is shown in Fig. 8. Drive power and forward power of the klystron can be measured via coupler 1 and 2, respectively. Input power and output power of the test stand can be measured from coupler 3 and 4 , respectively. The power gain of the klystron was measured around $45.6 \mathrm{~dB}$. Until now, we have some processing results for the first pair of the power couplers at the repetition period of $3 \mathrm{~S}$. The klystron was putting out around $65 \mathrm{~kW}$ of RF power before it began to saturate, as shown in Fig. $9.61 \mathrm{~kW}$ of input power and $54 \mathrm{~kW}$ of output power of the test stand were reached at all pulse lengths. Power reflection and transmission coefficients are shown in Fig. 10 for the pulse length of $1300 \mu \mathrm{s}$. The power reflection coefficient of the test stand is approximately $-18 \mathrm{~dB}$. The power transmission coefficient of the power coupler looks good. 
It is only $-0.6 \mathrm{~dB}$ for the test stand. It should be noted that the measurement error is $1 \%$ and attenuation of the rectangular waveguide is around $0.2 \mathrm{~dB}$. While running the test, the temperatures of the cold windows and vacuum pressures were almost kept constant, as shown in Fig. 11.

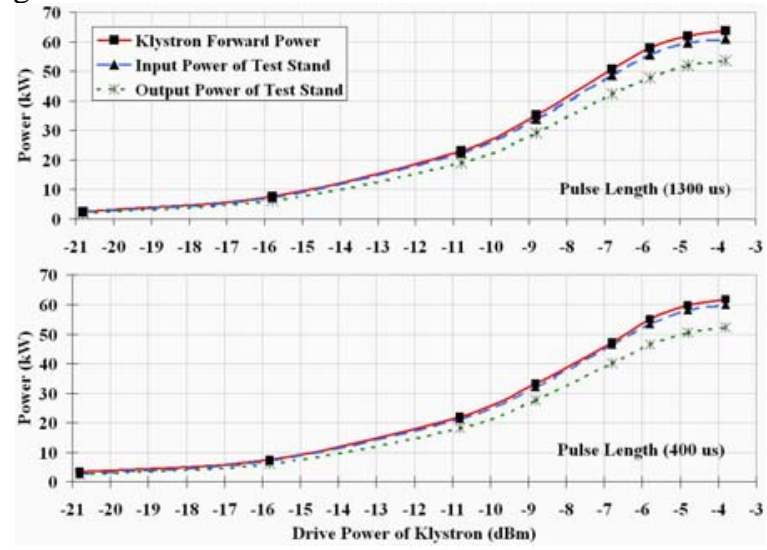

Figure 9: Measured powers versus the drive power of klystron.
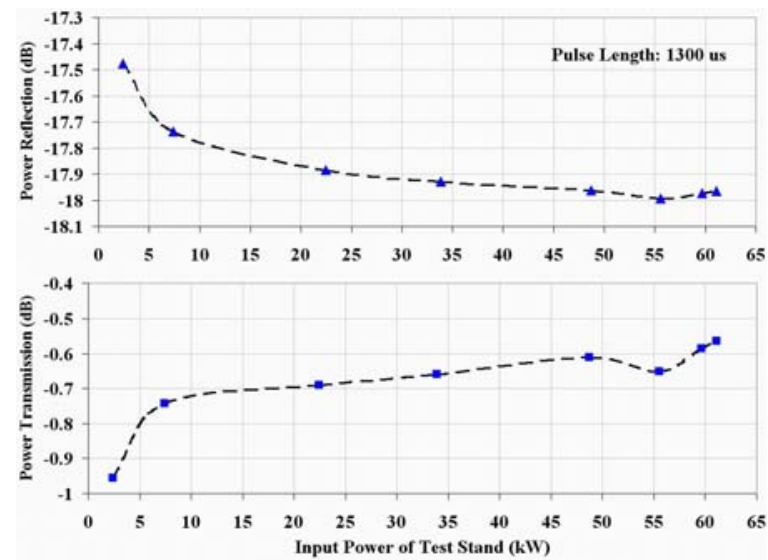

Figure 10: Power reflection and transmission coefficients of the test stand.

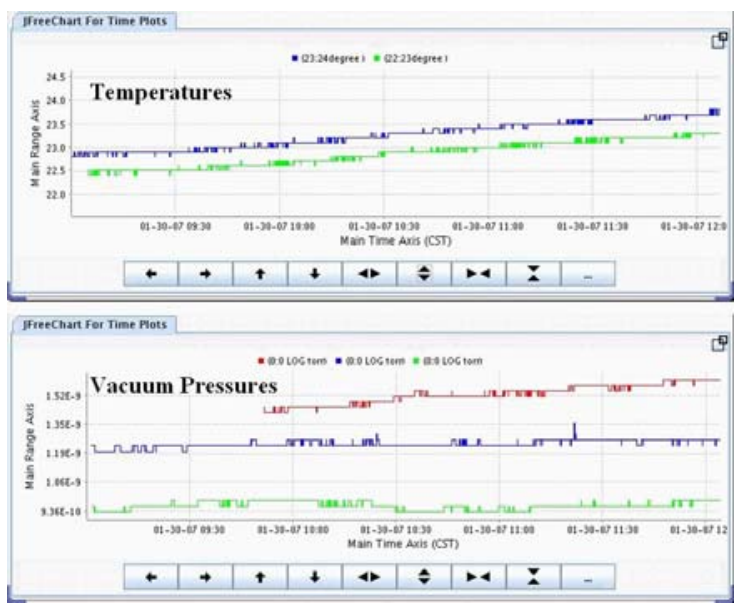

Figure 11: Temperature readings of the cold windows and vacuum pressures during the operation. Two vacuum gauges are installed in the warm areas of the couplers. One vacuum gauge is installed in the area of waveguide transition.

\section{CONCLUSION}

The power coupler designed in this way has a great RF performance and can meet the system strict requirements. Processing is very quiet without any problems or accidents till now. Most power is fed into the test stand with very low power reflection. More processing work will be continued with longer repetition rates and higher processing power levels. The power coupler integrated with a superconducting cavity will be installed in a horizontal test cryostat shown in Fig. 12 for the measurements of accelerating gradients, surface resistances, and quality factors in the next step. The design and commissioning work for the $3.9 \mathrm{GHz}$ cryogenic test is on schedule at Fermilab.

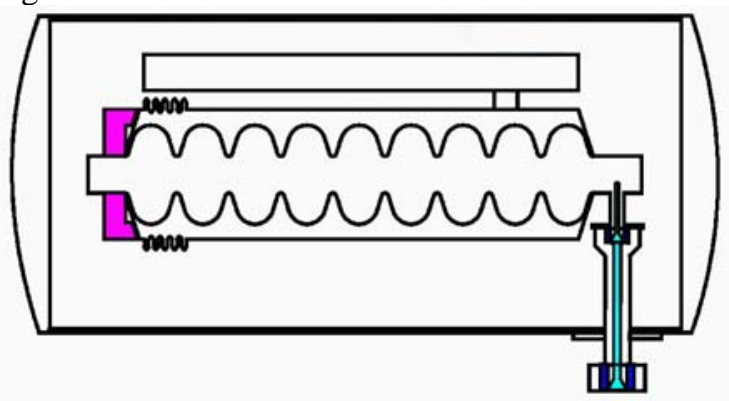

Figure 12: Model of the horizontal test cryostat.

\section{REFERENCES}

[1] N. Solyak et al., "Development of the Third Harmonic SC Cavity at Fermilab,” Proceedings of PAC 2003, Portland, Oregon, May 2003, p. 12131215.

[2] J. Li et al., "Simulations and Optimizations of a New Power Coupler for 3.9-GHz Superconducting Cavities at Fermilab,” Proceedings of LINAC 2006, Knoxville, TN, August 2006, p. 701-703.

[3] D. Kostin et al., "Status and Operating Experience of the TTF Coupler," Proceedings of LINAC 2004, Lubeck, Germany, August 2004, p.156-158. 